\title{
Inverse Estimation of Boundary Heat Flux for Heat Conduction Model
}

\author{
A. S. A. Alghamdi \\ Faculty of Engineering, Umm Al-Qura University, Makkah, Saudi Arabia \\ asaalghamdi@uqu.edu.sa
}

\begin{abstract}
Mathematical model of direct and inverse problems is developed for the flat plate probe which is subjected to timedependent heat flux at one end, while the other end is kept insulated. The direct solution, which is concerned with determination of the temperature distribution in the probe, is developed using an approach based on the method of variation of parameters. The direct solution is used to solve the inverse heat conduction problem (IHCP) for any assumed heat flux. In this paper, the piecewise linear interpolation model for heat flux is used. The inverse algorithm is developed using the Levenberg-Marquardt method. Different test cases for known heat flux profile are used to validate the inverse algorithm. A satisfactory agreement between exact and estimated heat flux profiles is achieved. The algorithm is used to estimate the heat flux for a flat plate probe developed at Ohio University.

Keywords: Direct problem, Inverse problem, Variation of parameters, IHCP
\end{abstract}

\section{Introduction}

An inverse problem in heat conduction consists of solving a direct problem and then finding some way to determine the inverse solution ${ }^{[1]}$. In inverse heat conduction problem ${ }^{[1]}$, the direct problem is used to determine the temperature distribution inside the solid body when certain initial and boundary conditions are given, such as temperature or heat flux, which are known as a function of time. In practice, the temperature in the solid can be monitored by sensors; however the surface heat flux cannot be determined experimentally. Theoretically, the surface heat flux 
history can be calculated from a set of temperature values. This type of problem is called "inverse heat conduction problem (IHCP)". The solution of the inverse heat conduction problem is difficult due to its sensitivity to measurements ${ }^{[2,3]}$.

Several numerical methods have been developed to solve the inverse problem due to its sensitivity to measurements. These methods are discussed in detail by M N Ozisiks et al. ${ }^{[1]}$ and J. Taler et al. ${ }^{[4]}$. Tervola ${ }^{[5]}$ developed a numerical method to determine thermal conductivity from measured temperature profiles. The boundary element method (BEM) was used by Lesnic et al. ${ }^{[6]}$ to determine the boundary conditions in a transient conduction problem where energies are specified in two areas of a one-dimensional slab. Tseng et al. ${ }^{[7]}$, Hunag et al. ${ }^{[8]}$, and Keanini ${ }^{[9]}$ described applications of IHCP to the manufacturing processes.

Estimation of heat flux from measured transient temperature history has received great interest during last three decades ${ }^{[1,4]}$. S. Abboudi et al. ${ }^{[10]}$ developed one dimensional heat conduction model to analyze numerically and experimentally transient heating of a flat specimen. The heat flux absorbed by the specimen was estimated using inverse analysis. Stolz ${ }^{[11]}$ developed a numerical solution for the inverse problem of heat conduction for some simple shapes. Alam et al. ${ }^{[12]}$, Kumar $^{[13]}$, and Zhong ${ }^{[14]}$ developed an inverse solution to determine the surface heat flux for quench probes in quenching experiment. These authors used a the polynomial model (representing heat flux with sixth degree polynomial) to solve the inverse problem for the probe ${ }^{[12,13]}$. They suggested that the results can be improved by representing the heat flux $q(t)$ by piecewise continuous polynomial of different degrees. The next step is to replace the polynomial model by cubic spline model ${ }^{[14]}$. The solution obtained by the procedure described is complicated and posed difficulties in the representation of heat flux in cubic spline. Also, the cubic spline exhibited sensitivity and stability problems. Therefore, a piecewise continuous straight line representation of $q(t)$ was suggested in order to improve the stability of the solution.

A classic problem in IHCP is the determination of surface heat flux in a one-dimensional slab. Quenching experiments are often carried out to determine the surface heat fluxes from experimental measurements of the temperature history. This is a typical inverse problem and therefore it 
is required to develop an algorithm with special considerations. This paper is concerned with several objectives. Firstly, inverse heat conduction model is developed based on the formulation of direct and inverse problem. Secondly the inverse algorithm is constructed and tested. Finally, numerical estimation of boundary heat flux for the flat plate probe is investigated based on experimental measurements of the temperature history obtained from Quenching experiment ${ }^{[15]}$.

\section{Mathematical Model}

\subsection{Direct Problem}

Consider a one-dimensional heat conduction problem through a uniform plate, as shown in Fig. 1. At one end, $x=0$, the surface is insulated, and the second end, $x=L$, is subjected to heat flux $q(L, t)$. This problem is described by the following set of equations:

$$
\begin{array}{ll}
\frac{\partial T(x, t)}{\partial t}=\alpha \frac{\partial^{2} T(x, t)}{\partial x^{2}}, & \text { in } 0<x<L, \text { for } t>0 \\
\frac{\partial T(0, t)}{\partial x}=0, & \text { at } x=0, \text { for } t>0 \\
k \frac{\partial T(L, t)}{\partial x}=q(t), & \text { at } x=L, \text { for } t>0 \\
T(x, 0)=T_{0}(x), & \text { for } t=0,0<x<L
\end{array}
$$

where

$$
\begin{array}{ll}
\alpha=\text { thermal diffusivity } & \begin{array}{l}
T=\text { temperature } \\
x=\text { space coordinate }
\end{array} \\
L=\text { thickness of plate } & q=\text { heat flux }
\end{array}
$$

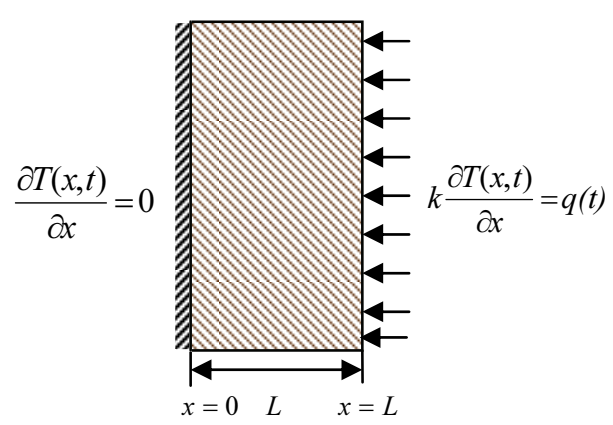

Fig. 1. One-dimensional flat plate probe with boundary conditions. 
For the case where the boundary condition at $x=L$, i.e., $q(t)$, the initial condition $T_{0}(x)$, and the thermo-physical properties $\alpha$, and $k$ are all specified, the problem given by Equation (1) is concerned with the determination of the temperature distribution $T(x, t)$ in the interior region of the solid as a function of time and position.

\subsection{Inverse Problem}

The inverse problem is concerned with the determination of the unknown function $q(t)$ at the surface $x=L$. To determine the heat flux $q(t)$, measured temperatures $T\left(x_{\text {meas }}, t_{i}\right) \equiv \stackrel{*}{T}_{i}$ are given at an interior point $x_{\text {meas }}$ at different times $t_{i}(i=1,2, \ldots, I)$, over a specified time interval $0<t \leq t_{f}$. The mathematical formulation of the inverse problem is given below:

$$
\begin{array}{cc}
\frac{\partial T(x, t)}{\partial t}=\alpha \frac{\partial^{2} T(x, t)}{\partial x^{2}}, & \text { in } 0<x<L, \text { for } 0<t \leq t_{f} \\
\frac{\partial T(0, t)}{\partial x}=0, & \text { at } x=0, \text { for } 0<t \leq t_{f} \\
k \frac{\partial T(L, t)}{\partial x}=q(t)=(\text { unknown }), \text { at } x=L, \text { for } 0<t \leq t_{f} \\
T(x, 0)=T_{0}(x), \quad \text { for } t=0,0<x<L
\end{array}
$$

and temperature measurements at an interior location $x_{\text {meas }}$ at different times $t_{i}$ are given by

$$
T\left(x_{\text {meas }}, t_{i}\right) \equiv T_{i}^{*} \text { at } x=x_{\text {meas }} \text { for } t=t_{i}(i=1,2, \ldots, I)
$$

In our problem, the boundary surface function heat flux $q(t)$ is unknown. Therefore, this version of the problem is referred to as a boundary inverse heat conduction problem. The main objective of the direct problem is to construct the temperature field $(T(x, t)$, the effect) in the plate, when all parameters (causes) are specified $\left(\alpha, k, q, T_{0}\right)$. On the other hand, the objective of the inverse problem is to estimate heat flux (the cause) from the knowledge of the measured temperature (the effect) at some specified section of the medium $\left(x_{\text {meas }}=0\right)$.

Alam et al. ${ }^{[12]}$ and Kumar ${ }^{[13]}$ developed an analytical solution, which consists of finding the temperature in a one-dimensional plate for a 
given heat flux which is assumed to be a polynomial function of time. It was suggested that the results can be improved by representing the $q(t)$ by piecewise continuous polynomial of different degrees. Zhong [14] conducted the study with a cubic spline function assigned to represent the heat flux. However, the heat flux and the heat transfer coefficients obtained from these approaches were not satisfactory.

\section{Solution of the Direct problem}

The method of variation of parameters, which is suitable for both steady-state and non-steady-state problems, is used to solve the direct heat conduction problem ${ }^{[16]}$. The problem will be decomposed to two parts: a problem formulation with prescribed initial condition and zero boundary conditions, and a second problem formulation with prescribed boundary conditions and zero initial condition. Since the problem is linear one can write:

$$
T(x, t)=u(x, t)+v(x, t)
$$

where $u(x, t)$ is the solution of

$$
\begin{aligned}
& \frac{\partial u(x, t)}{\partial t}=\alpha \frac{\partial^{2} u(x, t)}{\partial x^{2}}, \quad \text { in } 0<x<L \text {, for } t>0 \\
& \frac{\partial u(0, t)}{\partial x}=0, \quad \text { at } x=0 \text {, for } \quad t>0 \\
& \frac{\partial u(L, t)}{\partial x}=0, \quad \text { at } x=L \text {, for } t>0 \\
& T(x, 0)=T_{0}(x), \quad \text { for } t=0,0<x<L \\
& \frac{\partial v(x, t)}{\partial t}=\alpha \frac{\partial^{2} v(x, t)}{\partial x^{2}}, \quad \text { in } 0<x<L \text {, for } t>0 \\
& \frac{\partial v(0, t)}{\partial x}=0, \quad \text { at } x=0 \text {, for } t>0 \\
& k \frac{\partial v(L, t)}{\partial x}=q(t), \quad \text { at } x=L \text {, for } t>0 \\
& T(x, 0)=0, \quad \text { for } t=0,0<x<L
\end{aligned}
$$

The method of separation of variables can be used to find the solution of (5) 


$$
u(x, t)=\frac{C_{0}}{2}+\sum_{n=1}^{\infty} C_{n} \cos \left(\lambda_{n} x\right) \cdot \exp \left(-\alpha \lambda_{n}^{2} t\right)
$$

where

$$
C_{n}=\frac{2}{L} \int_{0}^{L} T_{0}(\xi) \cdot \cos \left(\lambda_{n} \xi\right) d \xi, \quad n=0,1,2, \ldots
$$

Making use of the solution of homogenous problem (5), we look for a solution in the form of a Fourier cosine series with time-dependent Fourier coefficients, that is

$$
v(x, t)=\frac{C_{0}(t)}{2}+\sum_{n=1}^{\infty} C_{n}(t) \cos \left(\lambda_{n} x\right)
$$

Using the orthogonality property of eigenfunction, differentiating with respect to time, integration along $\mathrm{x}$-axis and applying the boundary conditions leads to

$$
\frac{d C_{n}(t)}{d t}+\alpha \lambda_{n}^{2} C_{n}(t)=\frac{2 \alpha}{L K}(-1)^{n} \cdot q(t)
$$

The solution of the above first order linear ordinary differential equation subject to $C_{n}(0)=0$ is given by

$$
C_{n}(t)=\frac{2 \alpha}{L K}(-1)^{n} \int_{0}^{t} \exp \left(-\lambda_{n}^{2} \alpha(t-\tau)\right) \cdot q(\tau) d \tau
$$

By letting $n=0$, the above expression reduce to

$$
C_{0}(t)=\frac{2 \alpha}{L K} \int_{0}^{t} q(\tau) d \tau
$$

Using equations (11) and (12), the solution can be written as

$$
\begin{aligned}
T(x, t) & =\frac{1}{L} \int_{0}^{L} T_{0}(\xi) d \xi+\sum_{n=1}^{\infty}\left[\frac{2}{L} \int_{0}^{L} T_{0}(\xi) \cdot \cos \left(\lambda_{n} \xi\right) d \xi\right] \cdot \cos \left(\lambda_{n} x\right) \cdot \exp \left(-\alpha \lambda_{n}^{2} t\right) \\
& +\frac{\alpha}{L K} \int_{0}^{t} q(\tau) d \tau+\sum_{n=1}^{\infty}\left[\frac{2 \alpha}{L K}(-1)^{n} \int_{0}^{t} \exp \left(-\lambda_{n}^{2} \alpha(t-\tau)\right) \cdot q(\tau) d \tau\right] \cdot \cos \left(\lambda_{n} x\right)
\end{aligned}
$$

where

$$
\lambda_{n}=\frac{n \pi}{L}, \quad n=1,2,3, \ldots
$$

For a constant initial condition, i.e., $T(x, 0)=T_{0}$, the solution becomes 


$$
T(x, t)=T_{0}+\frac{\alpha}{L K} \int_{0}^{t} q(\tau) d \tau+\sum_{n=1}^{\infty}\left[\frac{2 \alpha}{L K}(-1)^{n} \int_{0}^{t} \exp \left(-\lambda_{n}^{2} \alpha(t-\tau)\right) q(\tau) d \tau\right] \cdot \cos \left(\lambda_{n} x\right)
$$

The solution (14) for $q(t)=$ constant agrees with solution presented by Beck et al. ${ }^{[2]}$.

\section{Inverse Problem Solution}

\subsection{Linear Spline Model}

In the previous approaches, the analytical solution was developed by representing the heat flux as a sixth-degree polynomial, Kumar ${ }^{[13]}$, and as a cubic spline by Zhong ${ }^{[14]}$. In this study, the heat flux $q(t)$ in the analytical solution is expressed as a linear spline function in $t$, i.e., as a piecewise straight line representation. Here the best fit straight line method is used to match the experimental data with such analytical data where the least square error between the linear spline and the experimental data is minimized.

In this method, a polynomial of degree 1 is the simplest polynomial to use which produces a polygonal path that consists of line segments that pass through the points. A point-slope formula for a line segment to represent this piecewise linear curve is given by the following expression

$$
q(t)=q_{i-1}+s_{i-1}\left(t-t_{i-1}\right), \text { where } s_{i-1}=\frac{q_{i}-q_{i-1}}{t_{i}-t_{i-1}}
$$

The resulting curve looks like a broken line, as seen in Fig. 2. The resulting linear spline function can be written in the form

$$
q(t)=\left\{\begin{array}{cc}
q_{0}+s_{0}\left(t-t_{0}\right) & \text { for } t \text { in }\left[t_{0}, t_{1}\right] \\
q_{1}+s_{1}\left(t-t_{1}\right) & \text { for } t \text { in }\left[t_{1}, t_{2}\right] \\
\vdots & \vdots \\
q_{i-1}+s_{i-1}\left(t-t_{i-1}\right) & \text { for } t \text { in }\left[t_{i-1}, t_{i}\right] \\
\vdots & \vdots \\
q_{N-1}+s_{N-1}\left(t-t_{N-1}\right) & \text { for } t \text { in }\left[t_{N-1}, t_{N}\right]
\end{array}\right.
$$

It is assumed that the abscissas are ordered $t_{0}<t_{1}<t_{2} \ldots<t_{N-1}<t_{N}$. For a fixed value of $t$, the interval $\left[t_{i-1}, t_{i}\right]$ 
containing $t$ can be found by successively computing the difference $t-t_{1}, \ldots, t-t_{i-1}, t-t_{i}$, such that $t-t_{i}<0$ for smallest $i$. Hence, for the described interval $t_{i-1} \leq t \leq t_{i}$, the value of the spline function $q(t)$ is

$$
q(t)=q_{i-1}+s_{i-1}\left(t-t_{i-1}\right) \quad \text { for } \quad t_{i-1} \leq t \leq t_{i} .
$$

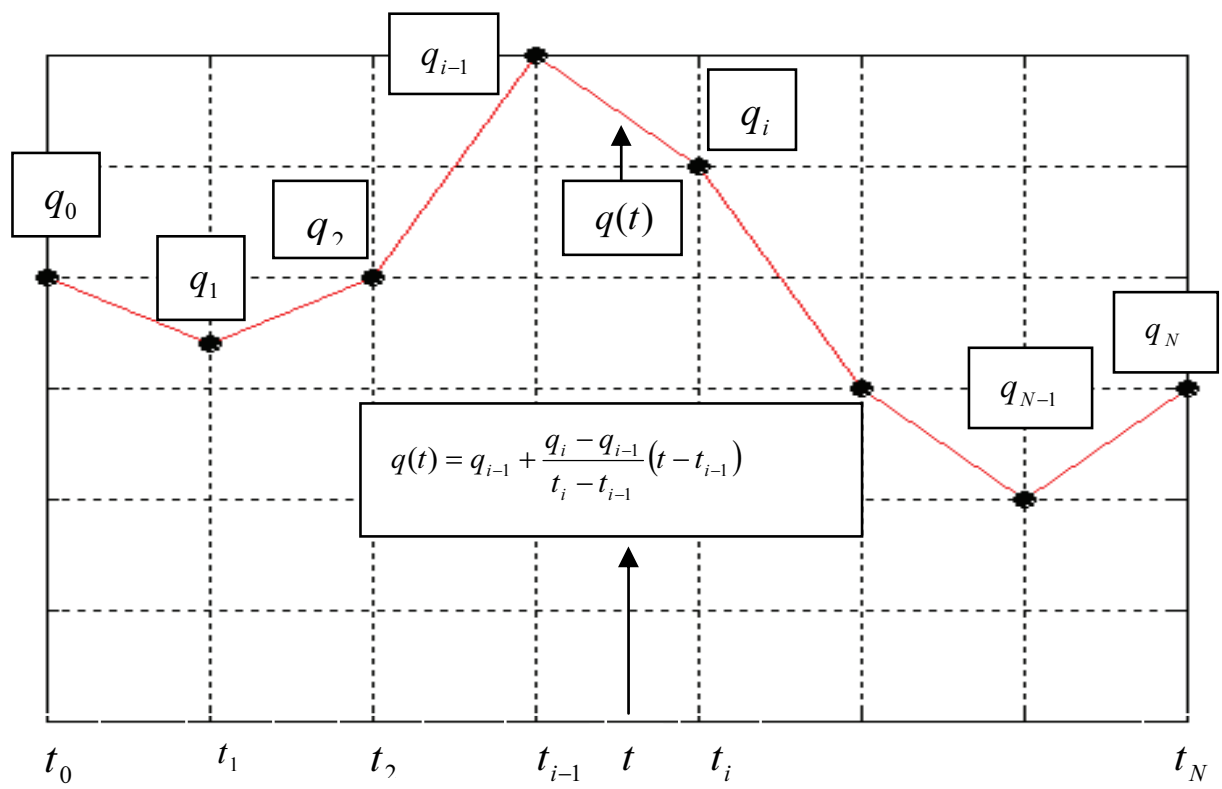

Fig. 2. Piecewise linear interpolation (a liner spline).

After determining the suitable interval for $t$ we can perform integration of $q(t)$ over the interval $\left[t_{0}, t\right]$ by dividing the interval into two subintervals $\left[t_{0}, t_{i-1}\right]$ and $\left[t_{i-1}, t\right]$. The first part of integration is the summation of the area of trapezoidal over the $j-1$ intervals. After the heat flux is calculated in the straight line form, the analytical temperature can be compared with the simulated data.

Substituting $q(t)$ in Equation (14), one can get

$$
\begin{aligned}
T(x, t)= & T_{0}+\frac{\alpha}{L K} \int_{0}^{t}\left[q_{i-1}+\frac{q_{i}-q_{i-1}}{t_{i}-t_{i-1}}\left(\tau-t_{i-1}\right)\right] d \tau \\
& +\sum_{n=1}^{\infty}\left\{\frac{2 \alpha}{L K}(-1)^{n} \int_{0}^{t} \exp \left(-\lambda_{n}^{2} \alpha(t-\tau)\right) \cdot\left[q_{i-1}+\frac{q_{i}-q_{i-1}}{t_{i}-t_{i-1}}\left(\tau-t_{i-1}\right)\right] d \tau\right\} \cos \left(\lambda_{n} x\right)
\end{aligned}
$$


Performing the integrations, the solution in terms of the piecewise straight lines heat flux becomes

$$
\begin{aligned}
& T(x, t)=T_{0}+\frac{\alpha}{L K}\left\{\begin{array}{l}
\sum_{i=1}^{j-1}\left[\frac{1}{2}\left(q_{i}+q_{i-1}\right)\left(t_{i}-t_{i-1}\right)\right] \\
+q_{j-1}\left(t-t_{j-1}\right)+\frac{1}{2} \frac{q_{j}-q_{j-1}}{t_{j}-t_{j-1}}\left(t-t_{j-1}\right)^{2}
\end{array}\right\}
\end{aligned}
$$

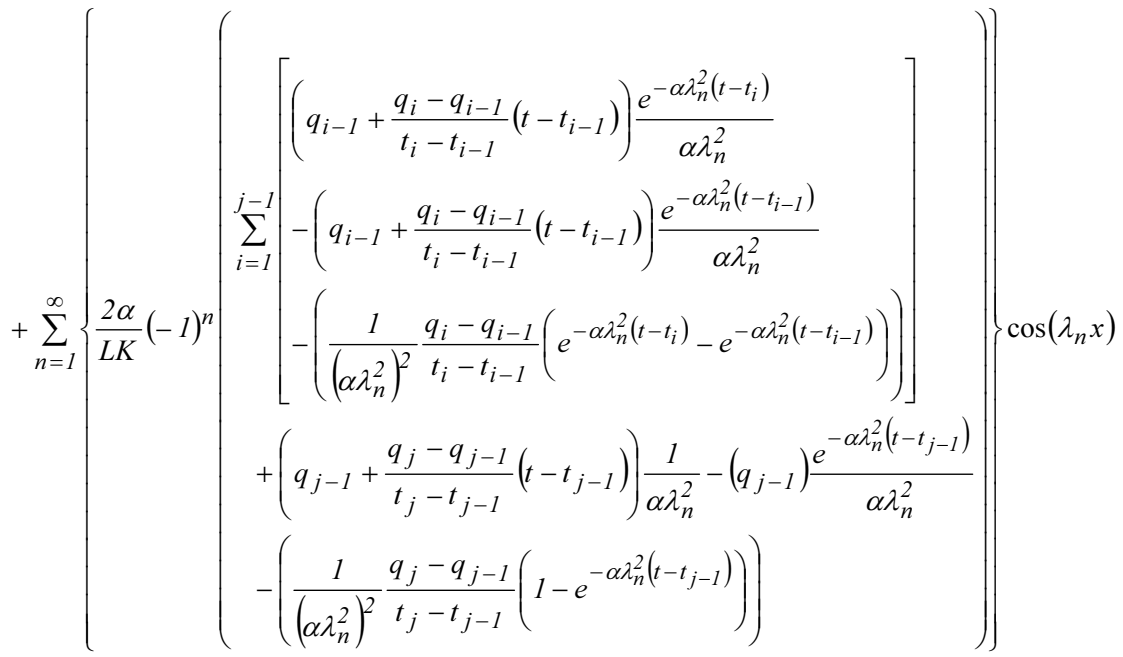

\subsection{Finite Difference Solution}

To check validation of the above solution (18), the direct problem (1) can be solved using a finite difference method. The method will start as follows:

Rewriting the partial differential equation in terms of finite difference approximations to the derivatives

$$
\frac{T_{j}^{n+1}-T_{j}^{n}}{\Delta t}=\alpha \frac{T_{j+1}^{n}-2 T_{j}^{n}+T_{j-1}^{n}}{\Delta x^{2}}
$$

Thus, if for certain $n$ we know the values of $T_{j}^{n}$ for all $\mathrm{j}$, ( initial condition $\left.T(x, 0)=T_{0}(x)\right)$, we can solve the equation above to find $T_{j}^{n+1}$ for each $j$ :

$$
T_{j}^{n+1}=T_{j}^{n}+\frac{\alpha \Delta t}{\Delta x^{2}}\left(T_{j+1}^{n}-2 T_{j}^{n}+T_{j-1}^{n}\right)=F_{0}\left(T_{j+1}^{n}+T_{j-1}^{n}\right)+\left(1-2 F_{0}\right) T_{j}^{n}
$$


where

Equation for the left boundary condition $\frac{\partial T(0, t)}{\partial x}=0$ :

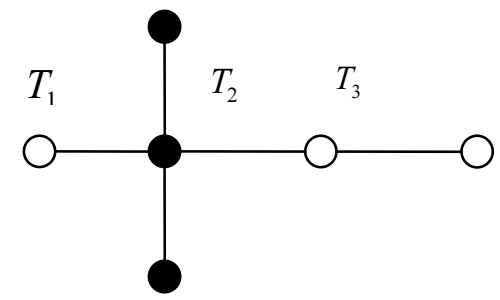

$$
\begin{aligned}
& T_{j}^{n+1}=F_{0}\left[2 T_{j-1}^{n}+T_{j}^{n}+T_{j}^{n}\right]+\left[1-4 F_{0}\right] T_{j}^{n} \\
& T_{j}^{n+1}=F_{0}\left[2 T_{j-1}^{n}\right]+\left[1-2 F_{0}\right] T_{j}^{n} \\
& T_{2}^{n+1}=2 F_{0} T_{3}^{n}+\left[1-2 F_{0}\right] T_{2}^{n}
\end{aligned}
$$

Equation for the right boundary condition $k \frac{\partial T(L, t)}{\partial x}=q(t)$

$$
\begin{aligned}
& k \frac{T_{j-1}^{n}-T_{j}^{n}}{\Delta x}+h\left(T_{\infty}-T_{j}^{n}\right)=\rho c \frac{\Delta x}{2} \frac{T_{j}^{n+1}-T_{j}^{n}}{\Delta t} \\
& k \frac{T_{j-1}^{n}-T_{j}^{n}}{\Delta x}+q\left(t^{n+1}\right)=\rho c \frac{\Delta x}{2} \frac{T_{j}^{n+1}-T_{j}^{n}}{\Delta t}
\end{aligned}
$$

Rearranging the above equation, we get

$$
\begin{aligned}
& T_{j}^{n+1}=F_{0}\left[2 T_{j-1}^{n}+\left(\frac{1}{F_{0}}\right) T_{j}^{n}+2 \frac{\Delta x}{k} q\left(t^{n+1}\right)\right] \\
& \text { where } \quad \alpha=\frac{k}{\rho c}, \quad F_{0}=\frac{\alpha \Delta t}{\Delta x^{2}}
\end{aligned}
$$

For stability purposes, we must have the following condition in the Fourier number

$$
F_{0}=\frac{\alpha \Delta t}{(\Delta x)^{2}} \leq \frac{1}{2}
$$

A finite difference program was written to compare numerical and analytical solutions for $q(t)=f(t)$. The temperature profile for both analytical and finite difference solutions was compared in Fig. 3. From analysis of Fig. 3 it follows that both solutions yield the same results. 

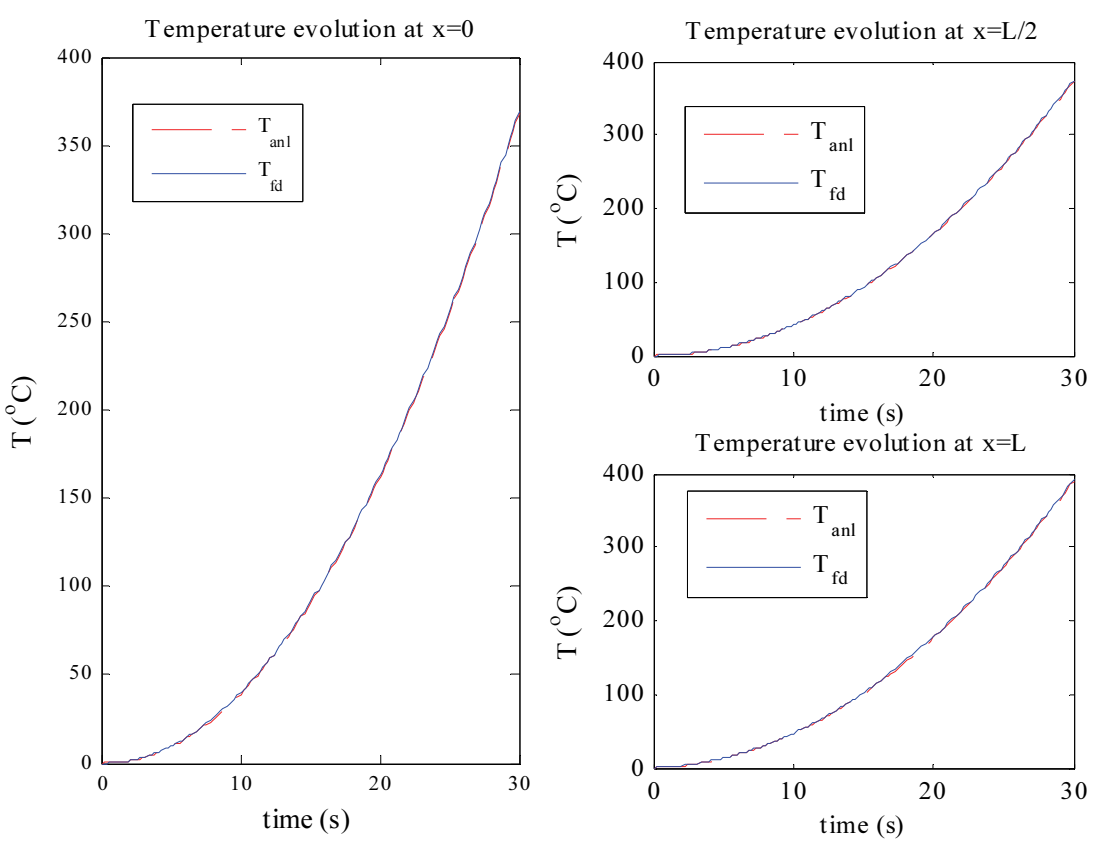

Fig. 3. Comparison between the analytical and the finite difference solutions for heat flux $q(t)=q_{0} t^{2}$

\subsection{MATLAB Code}

The Levenberg-Marquardt method is used to solve the inverse problem. It is an efficient method for solving linear problems that are illconditioned ${ }^{[17,18]}$. For the solution of the present inverse problem, we consider the unknown heat flux $q(t)$ to be expressed as linear spline function in $t$, i.e., as a piecewise straight line representation, with unknown parameters $q_{i}$.

The solution of the heat conduction problem (2) with $q(t)$ unknown parameterized as piecewise straight line function is an inverse heat conduction problem in which the parameters $q_{i}$ are to be estimated in equation (18). The solution of this inverse heat conduction problem for the estimation of the $N$ unknown parameters $q_{i}$, for $i=1,2, \ldots, N$, is based on the minimization of the ordinary least squares norm:

$$
S(q)=\sum_{i=1}^{I}\left[T_{i}^{*}-T_{i}(q)\right]^{2}
$$


where

$S=$ sum of squares error or objective function

$q^{T} \equiv\left[q_{1}, q_{2}, \ldots, q_{N}\right]=$ vector of unknown parameters

$T_{i}(q) \equiv T\left(q, t_{i}\right)=$ estimated temperature at time $t_{i}$

$\stackrel{*}{T}_{i} \equiv \stackrel{*}{T}\left(t_{i}\right)=$ measured temperature at $t_{i}$

$N=$ total number of the unknown parameters

$I=$ total number of measurements, where $I \geq N$

The estimated temperatures $T_{i}(q)$ are obtained from the solution of the direct problem at the measurement location, $x_{\text {meas }}$, by using the estimated unknown parameters $q_{i}, i=1,2, \ldots, N$. The unknown vector $q^{T} \equiv\left[q_{1}, q_{2}, \ldots, q_{N}\right]$ is determined by minimizing $S(q)$ using the iterative procedure in the Levenberg-Marquardt method ${ }^{[17,18]}$.

After defining the direct and inverse formulations, the Matlab code is written to solve the inverse problem with the help of optimization tool box ${ }^{[19]}$ which impelements the criteria, and the Levenberg-Marquardt method. The iterative procedure, the stopping criteria, and the computational algorithm were chosen based on the Levenberg-Marquardt Matlab function capability.

\section{Test Cases: Inverse Solution for Known Heat Flux Profile}

In this section, two test cases are discussed. One test case is for a heat flux that increases in a linear fashion at time. Exact values of the simulated temperature history are used. The second case is for a heat flux which varies at time in a triangular fashion; for this case both exact temperatures and temperatures with random errors are used.

For the time interval $0<t \leq 120$, we consider 100 transient measurements of a single sensor located at $x_{\text {meas }}=0$.

\subsection{Simulated Measurements}

Simulated measurements are obtained from the solution of the direct problem at sensor location by using prior prescribed values for the unknown parameters $q_{i}$ of heat flux $q(t)$. 
For example, if we consider that we can represent the heat flux with 10 parameters, i.e.,

$$
q(t)=q_{i-1}+\frac{q_{i}-q_{i-1}}{t_{i}-t_{i-1}}\left(t-t_{i-1}\right),
$$

with $q=\left[q_{1}, q_{2}, \ldots, q_{10}\right]$ at $t_{i}=t_{1}, t_{2}, \ldots, t_{10}$, for $N=10$.

By using the known heat flux described above, the solution (18) of the direct problem at the measurement location $x_{\text {meas }}=0$ provides the exact (errorless) measurements $\stackrel{*}{T}_{e x}\left(t_{i}\right)$, for $i=1, \ldots, I$. Measurements containing random errors are simulated by adding an error term to $T_{e x}^{*}\left(t_{i}\right)$ in the form ${ }^{[1]}$

$$
\text { T }\left(t_{i}\right)=T_{e x}^{*}\left(t_{i}\right)+\varpi \sigma
$$

where

$T\left(t_{i}\right)=$ simulated measurements containing random errors

*

$T_{\text {ex }}\left(t_{i}\right)=$ exact (errorless) simulated measurements

$\sigma=$ standard deviation of the measurements errors

$\varpi=$ random variable with normal distribution, zero mean and unitary standard deviation, for the $99 \%$ confidence level we have $-2.576<\varpi<2.576$.

With use of such simulated measurements as the input data for the inverse analysis, we expect the code to return the same values of parameters used to find the direct solution.

\subsection{Case One: Linear Increase in Heat Flux}

The surface heat flux $q(t)$ increases linearly in time in the form $q(t)=q_{0} t$ over the interval $0<t \leq t_{f}$. The results of the inverse calculation for case $\sigma=0$ are shown in Fig. 4. A comparison of the exact simulated and estimated values of the temperatures profile at insulated surface of probe shown in the figure reveals that they are in excellent agreement. Also, it is clear from the figure the recovered heat flux coincides with the exact heat flux. To test the code accuracy, a simulated measurement containing random errors was used. Figure 5 compares the heat flux profiles for both cases to the exact profile, a 
satisfactory result for the case of perturbed measurements is achieved. The results obtained in the figure show that increasing the measurement errors decrease the accuracy of the inverse algorithm.
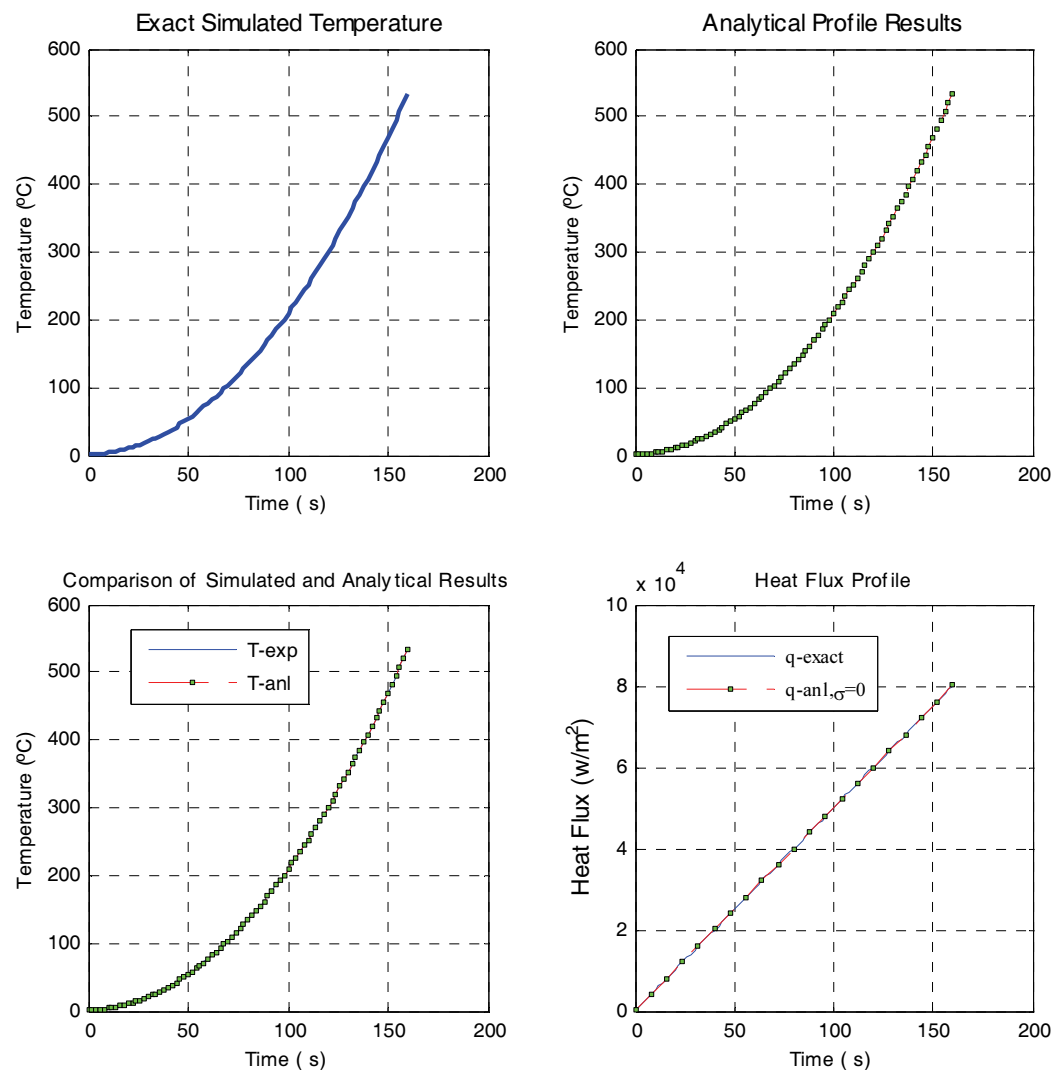

Fig. 4. Results from IHCP algorithm using time-varying heat flux in the form $q(t)=q_{0} t$.

\subsection{Case Two: Triangular Heat Flux}

In this case the heat flux varies in time in a triangular fashion. To form the heat flux profile, we let the surface heat flux $q(t)$ increases linearly with time for $t$ between zero and 60, and for $t>60$ the flux decreases linearly to zero at $t=120$ and remains zero thereafter. Mathematically, it is in the form: 


$$
q(t)=\left\{\begin{array}{cc}
q_{0} t_{z} & 0 \leq t \leq 60 \\
-\left(q_{0} / 60\right)(t-60), & 60<t \leq 120 \\
0_{r} & 120<t \leq 160
\end{array}\right.
$$

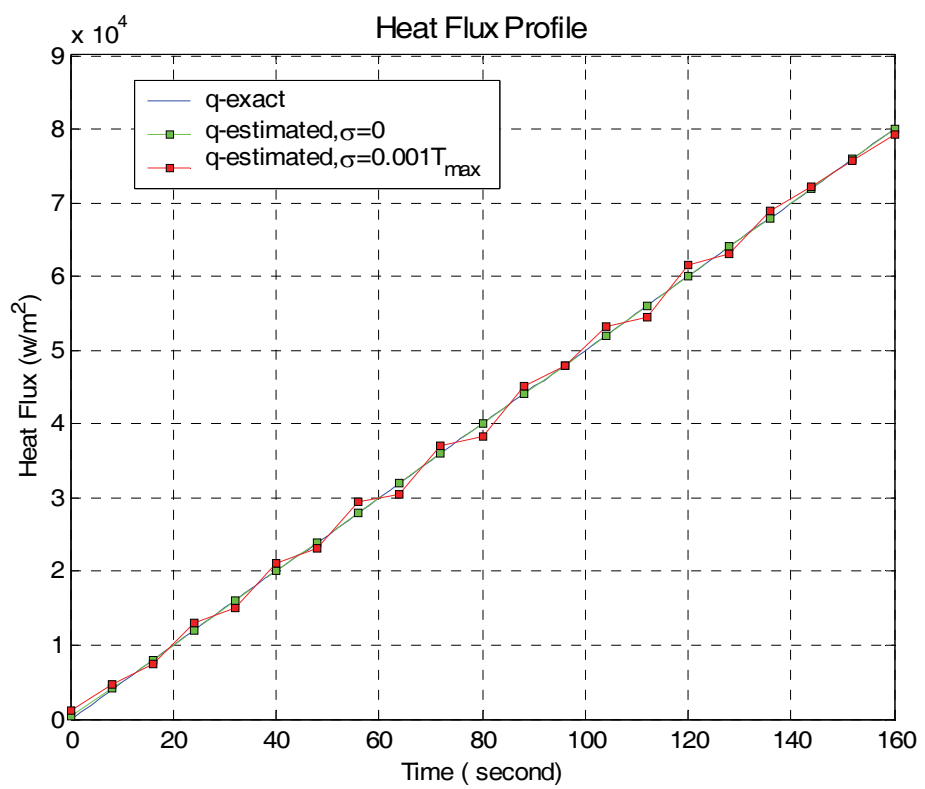

Fig. 5. The estimation of the time-varying boundary heat flux as straight line fashion.

Using $q(t)=q_{0} t$ and with help of superposition one can find the direct solution to this problem. Solving the inverse problem using the exact simulated result shows that the triangular heat flux profile matches the exact profile quite well, as well as the temperature profiles, as indicated in Fig. 6. For the case of simulated measurements containing random errors, Fig. 7 shows that the result is in a good agreement with the exact profile. As expected it is easier to recover a continuous function such as $q(t)=q_{0} t$, than a discontinuous function like triangular heat flux given by Equation (30). To over this problem, smaller step size $\Delta t$ and suitable measurements locations were used. Figure 7 shows that the sharp corner can be predicted very well by reduction of step size $\Delta t$.

\section{Application}

An industrial problem such as thermal treatment of metal alloys can be modeled as an inverse problem. Quenching is a particular type of 
thermal treatment process that involves rapid cooling of metal alloys for the purpose of hardening. Experimental measurements of temperature history of quenching experiments can be used to determine the surface heat fluxes. The developed model was used to estimate the heat flux for the quenching experiment developed at Ohio University ${ }^{[20]}$. The developed algorithm was used to solve this problem ${ }^{[15]}$, but for the sake of completeness, the description of the experiments and the results will be presented.
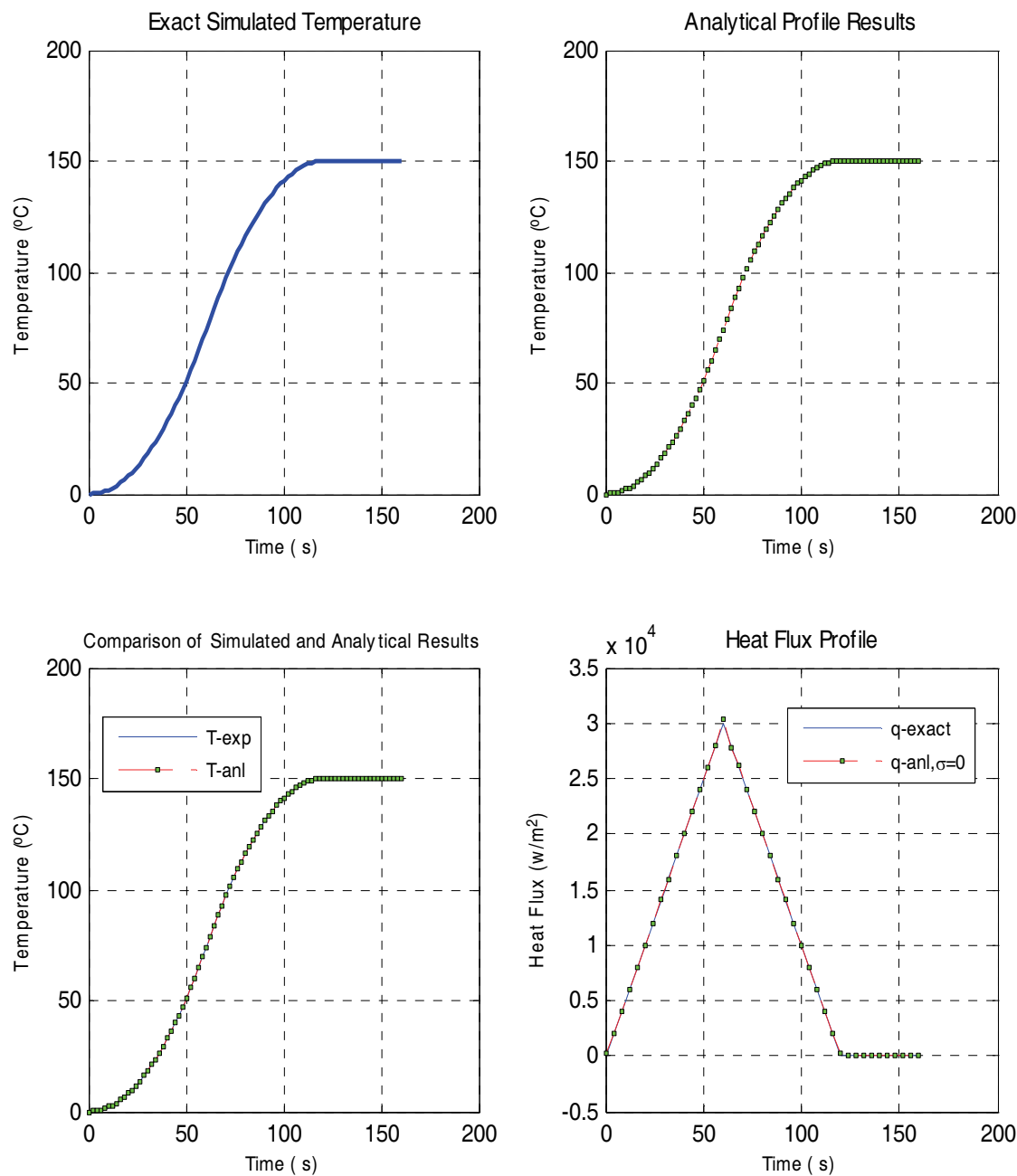

Fig. 6. Results from IHCP algorithm using time-varying heat flux in the form of triangular function. 


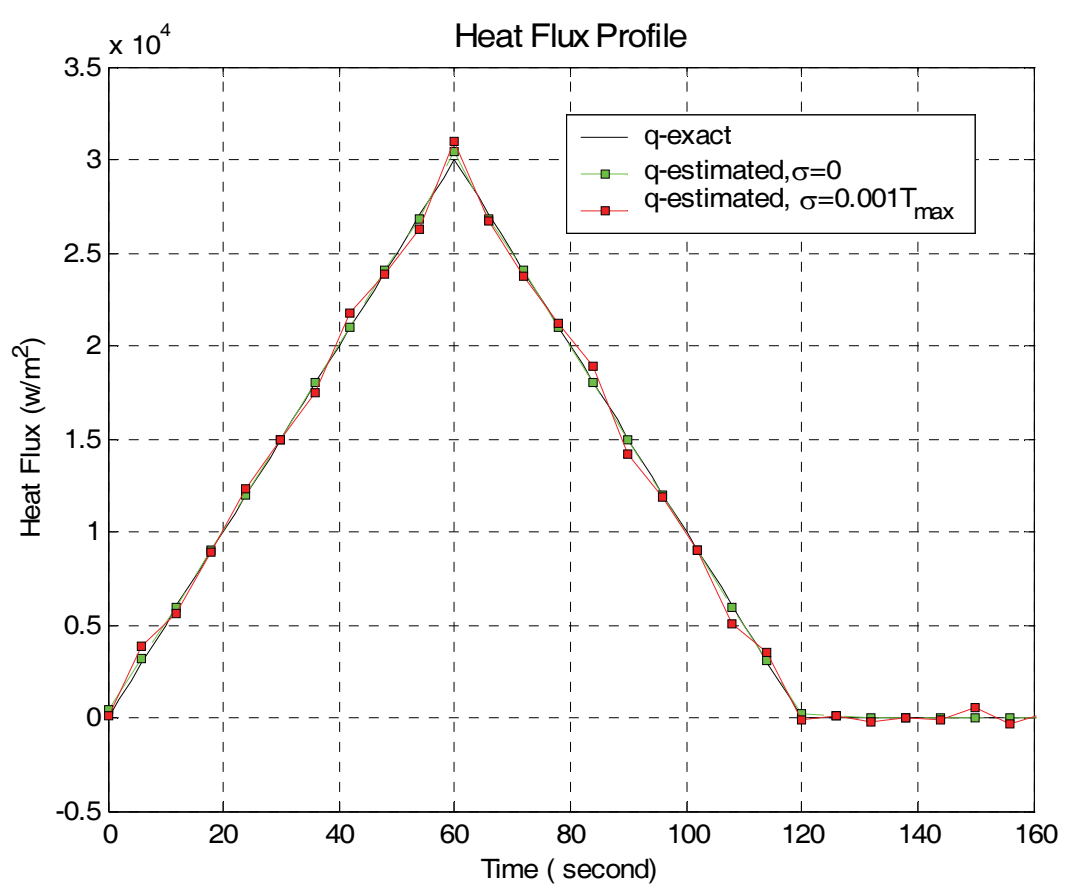

Fig. 7. The estimation of the time-varying boundary heat flux as triangular function fashion.

Experiments were conducted with a stainless steel probe in the shape of a rectangular box as shown in Fig. 8a. The sides of the box are approximated by, with each wall $6 \mathrm{~mm}$ thick ${ }^{[20]}$. The experimental results of temperature data versus time is given in Fig. $8 \mathrm{~b}$ for the thermocouple which is attached to the inside surface (at the center) of the wall of the probe. Since the wall thickness $6 \mathrm{~mm}$ is much smaller than the wall dimensions, the one-dimensional solution is valid for this wall of the probe. The temperature data collected from the quench probe is shown in Fig. 8b. The period corresponds to the air cooling of the probe during transport from oven to the quenching oven is removed.

Results from IHCP algorithm are presented in Fig. 9. In order to improve the accuracy of algorithm, the time step is significantly reduced. The experimental and analytical temperatures are compared in Fig. 9. It can be seen that the two temperature profiles match quite well. This indicates that the inverse solution is accurate, and it can be used to find the heat flux. Based on the analytical profile, the heat flux is then estimated based on Equations (18) and (28). The results are also shown in 
Fig. 9. The heat flux values from the plate are very high initially, as expected and gradually decays to zero. The heat flux is seen to have a maximum value of $450 \mathrm{kw} / \mathrm{m}^{2}$.

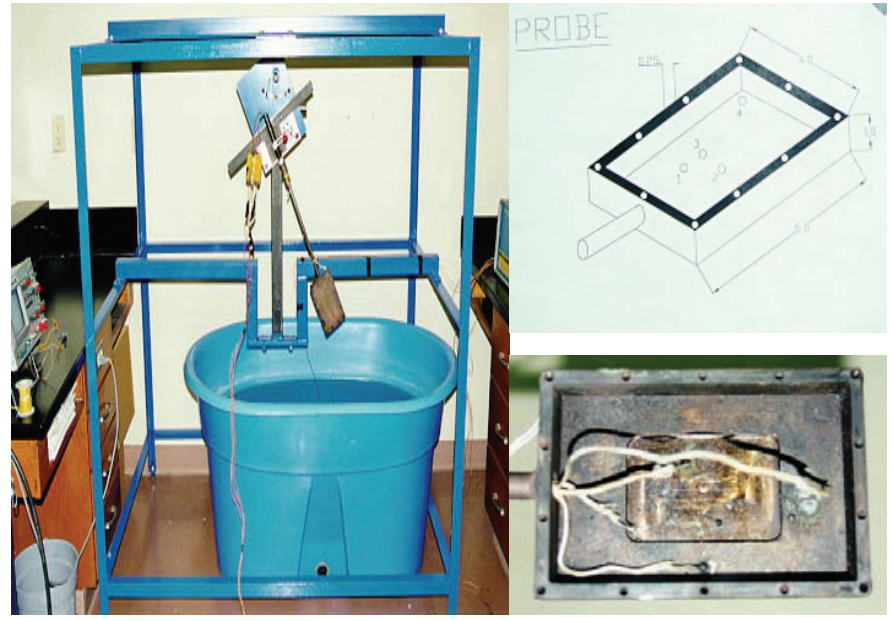

a) Picture of the quenching system.

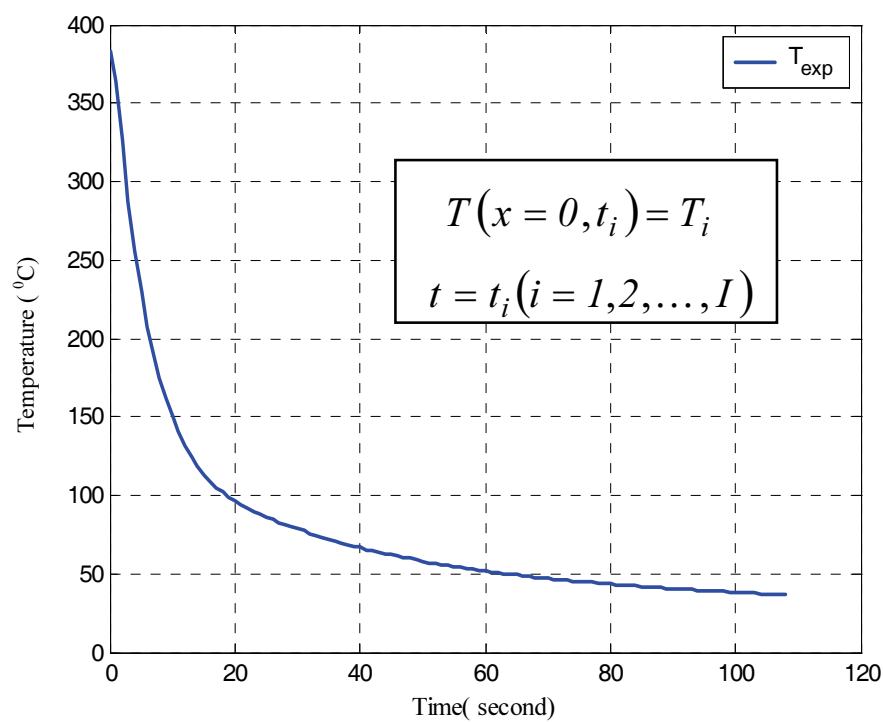

b) Cooling curve for the probe.

Fig. 8. Quenching experiment: a)- Picture of the quenching system , b)- Cooling curve for the probe (source: Ohio University ${ }^{[14,20]}$ ) 

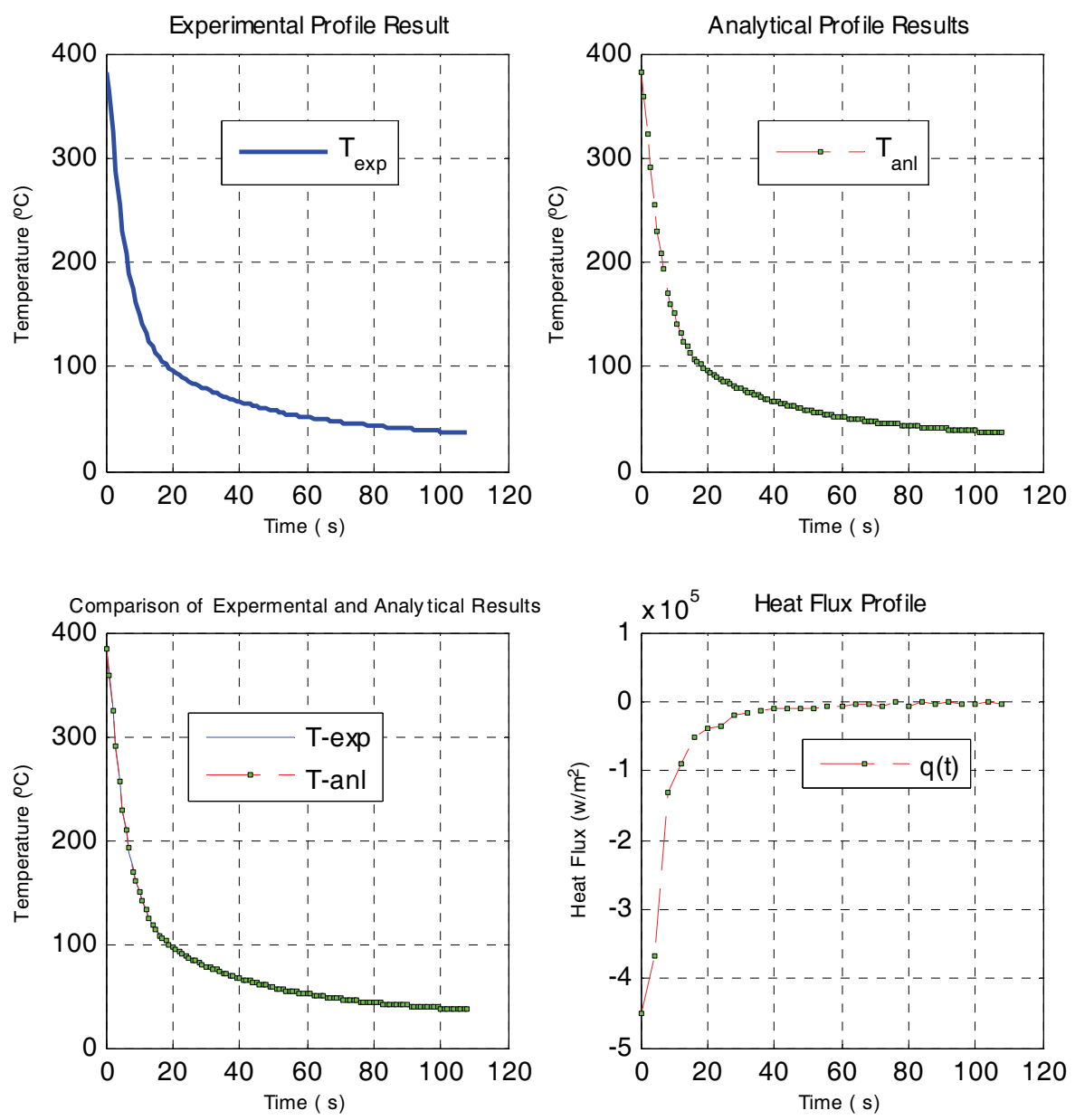

Fig. 9. Results from IHCP algorithm using experimental data from quench probe ${ }^{[15]}$.

Previous studies of this inverse heat conduction problem, which included the $6^{\text {th }}$ degree polynomial and cubic spline, have some limitations ${ }^{[12,14]}$. The cubic spline exhibits sensitivity of heat flux to input data. When a cubic spline curve is used, the heat flux curve is assumed to be smooth so that the first derivative is continuous at the interface between the intervals. Consequentially, this requirement produces variations in the heat flux curve similar to sinusoidal curves. Using a piecewise continuous straight line, on the other hand, does not require a heat flux curve to be smooth. Therefore, in order to improve the 
stability of the solution, a piecewise continuous straight line representation of $q(t)$ has been used.

\section{Conclusions}

An analytical solution of the direct problem, which consists of determining the temperature distribution in a one-dimensional uniform plate for a given time-dependent heat flux boundary condition at one end and the other end kept insulated, was developed for general form of heat flux. The direct solution is determined by an approach based on the method of variation of parameters. The solution is identical to the solution found by Beck et al. ${ }^{[2]}$ for constant heat flux.

The analytical solution is developed based on a piecewise linear representation of the heat flux and tested for different profiles. The solution shows an excellent agreement with finite difference solution. The piecewise linear interpolation model for heat flux is constructed to be used in the inverse solution. The heat flux profile or values were then found by employing the Levenberg-Marquardt method to fit the analytical solution based on simulated data. Then the analytical solution of the temperature profile over the plate is found based on this heat flux. Comparing temperatures history calculated by the inverse algorithm with that found by using known heat flux profile (simulated data) shows that the two solutions are in excellent agreement.

As an industrial problem, the algorithm was applied to experimental data obtained from a quenching experiment developed at Ohio University. The heat flux history during the quenching process is found and the theoretical temperature curve obtained from the analytical solution is compared with experimental results. The two temperature solutions show very satisfactory agreement ${ }^{[15]}$.

\section{Nomenclature}

\section{Acronyms}

IHCP

Inverse heat conduction problem

\section{English Symbols}

$\begin{array}{lll}k & \text { thermal conductivity } & {[\mathrm{W} / \mathrm{m} . K]} \\ L & \text { thickness of plate } & {[\mathrm{m}]} \\ q & \text { heat flux } & {\left[\mathrm{W} / \mathrm{m}^{2}\right]}\end{array}$




$\begin{array}{lll}S & \text { sum of squares error or objective function } & {[-]} \\ T & \text { temperature } & {\left[{ }^{0} \mathrm{C}\right]} \\ t & \text { time } & {[\mathrm{s}]} \\ x & \text { space coordinate } & {[\mathrm{m}]} \\ u & \text { temperature } & {\left[{ }^{0} \mathrm{C}\right]} \\ N & \text { total number of the unknown parameters } & {[-]} \\ I & \text { total number of measurements } & {[-]} \\ \text { Greek Symbols } & & \\ \alpha & & \\ v & \text { thermal diffusivity } & {\left[\mathrm{m}^{2} / \mathrm{s}\right]} \\ \lambda & \text { temperature } & {\left[{ }^{0} \mathrm{C}\right]} \\ \tau & \text { eigenvalues } & {[-]} \\ \zeta & \text { dummy variable } & {[-]} \\ & \text { dummy variable } & {[-]}\end{array}$

\section{Subscripts}

$i$
$n$
0
$f$
meas

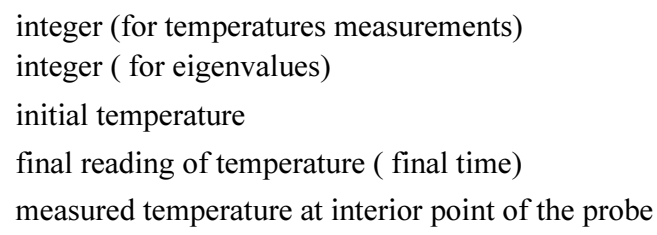

\section{Superscripts}

$\begin{array}{ll}* & \text { measured temperature at } t_{i} \\ n & \text { integer (for eigenvalues) } \\ T & \text { transpose }\end{array}$

\section{References}

[1] Ozisik, M. N. and Orlande, R. B. H., Inverse Heat Transfer, Taylor and Francis, New York (2000).

[2] Beck, J. V., Blackwell, B. and St. Clair, C. R., Inverse Heat Conduction, III Posed Problems, A Wiley Interscience Publication, New York (1985).

[3] Beck, J., Blackwell, V. B. and Sheikh-Haji, A., Comparison of Some Inverse Heat Condition Methods Using Experimental Data, Int. J. Heat Mass Transfer, 39(17): 36493657 (1996).

[4] Taler, J. and Duda, P., Solving Direct and Inverse Heat Conduction Problems, Springer, Berlin (2006).

[5] Tervola, P., A Method to Determine the Thermal Conductivity from Measured Temperature Profiles, Int. J. Heat Mass Transfer, 32:1425-1430 (1989).

[6] Lesnic, D., Elliott, L. and Ingham, D. B., The Solution of an Inverse Heat Conduction Problem Subject to the Specification of Energies", Int. J. Heat Mass Transfer, 41: 25-32 (1998). 
[7] Tseng, A. A., Chang, J. G., Raudensky, M. and Horsky, J., An Inverse Finite Element Evaluation of Roll Cooling in Hot Rolling of Steels, Journal of Material Processing and Manufacture Science, 3: 387-408 (1995).

[8] Huang, C. H., Ju, T. M. and Tseng, A. A., The Estimation of Surface Thermal Behavior of the Working Roll in Hot Rolling Process, Int. J. Heat Mass Transfer, 38: 1019-1031 (1995).

[9] Keanini, R. G., Inverse Estimation of Surface Heat Flux Distributions During High Speed Rolling Using Remote Thermal Measurements, Int. J. Heat Mass Transfer, 41: 275-285 (1998).

[10] S. Abboudi, E. Artiouknine and H. Riad, Computational and Experimental Estimation of Boundary Conditions for a Flat Specimen, Preliminary program inverse problems in engineering iii, Port Ludlow Resort \& Conference Center, 200 Olympic Place, Port Ludlow, WA 98365 (1999).

[11] Stolz, G., Jr., Numerical Solutions to an Inverse Problem of Heat Conduction for Simple Shapes, J. Heat Transfer, 82:20-26 (1960).

[12] Alam, M. K., Pasic, H., Anugarthi, K. and Zhong, R., Determination of Surface Heat Flux in Quenching, ASME IMECE Proceedings, Nashville, November (1999).

[13] Kumar, Analytical Solution for Inverse Heat Conduction Problem, M.S. Thesis, Ohio University (1998).

[14] Zhong, R., Inverse Algorithm for Determination of Heat Flux, M.S. Thesis, Ohio University (1999).

[15] Alghamdi, A. S. A., and Alam, M. K., Inverse heat transfer solution for the flat plate probe, Proceedings of the $3^{r d}$ BSME-ASME International Conference on Thermal Engineering, Dhaka, Bangladesh (2006).

[16] Poulikakos, D., Conduction Heat Transfer, Prentice-Hall, Inc (1994).

[17] Levenberg, K., A Method for the Solution of Certain Non-linear Problems in Least Squares, Quart. Appl. Math., 2:164-168 (1944).

[18] Mrquardt, D. W., An Algorithm for Least Squares Estimation of Nonlinear Parameters, $J$. Soc. Ind. Appl. Math, 11: 431-441 (1963).

[19] Chong, K. P. E. and Zak, H. S., An Introduction to Optimization, Prentice-Hall, Inc (1996).

[20] Zajc, D., Experimental Study of a Quench Process, M.S. Thesis, Ohio University (1998). 


\section{التقدير العكسي للفيض الحراري المتاخم لنموذج توصيل الحرارة}

\section{عبدالمجيد سعيد علي الغامدي}

كلبة الهندة، جامعة أم القرى، مكة المكرمة، المدلكة العربية السعودية

المستخلص: في هذا البحث تم تصميم نموذج رياضي مباشر

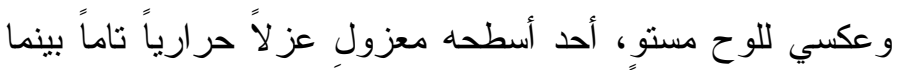
الآخر معرض لفيض حراري متغير مع الزمن. نم إيجاد الحل

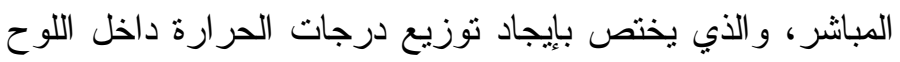
المستوي الأسطح، بطريقة تعتمد على طريقة اختلاف البار امتر ات. وكان الهذف من إيجاد هذا الحل هو حل النموذج الحراري العكسي (IHCP) الفيض الحر اري بنموذج رياضي خطي مشكل بقطع متصلة. ولحل

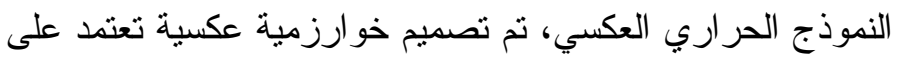

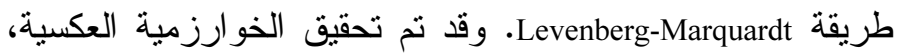
بإجر اء عدة اختبار ات للبرنامج المصمح باستخدام دوال معروفة

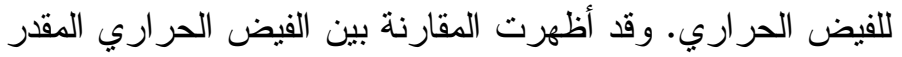
و المعروف نتيجة مرضية لجميع الحالات. كما استخدم هذا البرنامج

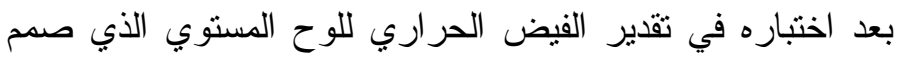

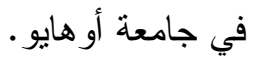

